\section{Comparison of needle aspiration and vacuum-assisted biopsy in the ultrasound-guided drainage of lactational breast abscesses}

\author{
Yun-Dan Kang ${ }^{1}$, You Me Kim² \\ Departments of ${ }^{1}$ Obstetrics and Gynecology and ${ }^{2}$ Diagnostic Radiology, Dankook University \\ College of Medicine, Cheonan, Korea
}

Purpose: The purpose of this study was to compare needle aspiration and vacuum-assisted biopsy in the ultrasound-guided treatment of lactational breast abscesses.

Methods: Between January 2005 and December 2014, a total of 74 patients presented with lactational breast abscesses. Thirty of these patients underwent treatment with antibiotics alone, while the remaining 44 lactating women with breast abscesses were treated with needle aspiration $(n=25)$ or vacuum-assisted biopsy $(n=19)$. Age, duration of lactation, abscess diameter, pus culture results, the number of interventions, the healing time, and the cure rate were reviewed and compared between these two groups. The Student's t test and the chi-square test were used to compare the variables.

Results: No significant difference was found in the cure rate between the needle aspiration group $(22 / 25,88 \%)$ and the vacuum-assisted biopsy group $(18 / 19,94.7 \%)(P=0.441)$. However, the mean healing time was significantly shorter in the vacuum-assisted biopsy group (6.7 days) than in the needle aspiration group ( 9.0 days) $(P=0.001)$.

Conclusion: Vacuum-assisted biopsy is a viable option for the management of lactational breast abscesses and was found to lead to a shorter healing time than needle aspiration. However, further study is necessary to establish the clinical efficacy of vacuum-assisted biopsy in the management of lactational breast abscesses.

Keywords: Ultrasonography; Breast; Biopsy, needle; Abscess; Image-guided biopsy;

Aspiration, vacuum-assisted

\section{Introduction}

Breast infections are a common problem in lactating women, with manifestations that range from mastitis to abscess formation. A breast abscess is defined as a localized infection with accumulation of fluid in the breast tissue. Breast abscesses can be classified as puerperal and nonpuerperal, and the nonpuerperal types are subclassified as peripheral or central periareolar [1]. Traditionally, breast abscesses were managed via incision and drainage with antibiotic therapy, but this treatment strategy

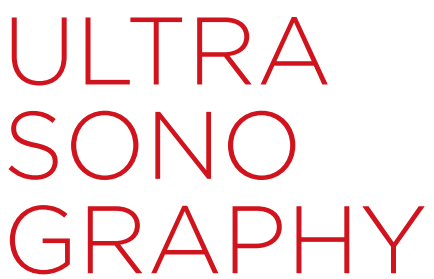

ORIGINAL ARTICLE

http://dx.doi.org/10.14366/usg. 15041 pISSN: 2288-5919 - elSSN: 2288-5943 Ultrasonography 2016;35:148-152

Received: July 27, 2015

Revised: September 17, 2015

Accepted: December 8, 2015

Correspondence to:

You Me Kim, MD, Department of

Diagnostic Radiology, Dankook

University College of Medicine, 119

Dandae-ro, Dongnam-gu, Cheonan

31116, Korea

Tel. +82-41-550-3967

Fax. +82-41-552-9674

E-mail: sirenos@hanmail.net

This is an Open Access article distributed under the terms of the Creative Commons Attribution NonCommercial License (http://creativecommons.org/ licenses/by-nc/3.0/) which permits unrestricted noncommercial use, distribution, and reproduction in any medium, provided the original work is properly cited.

Copyright $(2016$ Korean Society of Ultrasound in Medicine (KSUM)

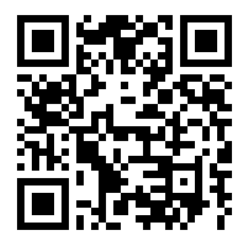

How to cite this article:

Kang YD, Kim YM. Comparison of needle aspiration and vacuum-assisted biopsy in the ultrasound-guided drainage of lactational breast abscesses. Ultrasonography. 2016 Apr;35(2):148-152. 
is associated with a prolonged healing time, regular dressings, and unsatisfactory cosmetic outcomes [2]. A number of studies have recently reported that breast abscesses can be treated by ultrasound-guided catheter placement or needle aspiration $[3,4]$. Needle aspiration has therefore supplanted incision and drainage as the first-line management strategy in many clinical contexts [3]. However, needle aspiration has some limitations for large abscesses or abscesses with thick material [5]. Ultrasound-guided vacuumassisted biopsy (VAB) is as an efficient tool for the diagnosis of breast lesions and has clear advantages over fine needle aspiration cytology and core biopsy in certain situations [6]. Recently, VAB has been used as in the management of breast abscesses $[7,8]$; therefore, the goal of this study was to compare the effectiveness of $V A B$ and needle aspiration in the treatment of lactational breast abscesses.

\section{Materials and Methods}

This retrospective study was approved by the Institutional Review Board of Dankook University Hospital. The medical database at Dankook University Hospital was queried from January 1, 2005 to December 31, 2014 to identify all patients with a diagnosis of a breast abscess. During that period, 342 women who were clinically suspected to have a breast abscess underwent breast ultrasonography. Sonographic criteria suggestive of a breast abscess include hypoechoic collection, being mostly multiloculated, the absence of vascularity within the collection, acoustic enhancement due to fluid content, and an echogenic vascular rim. The

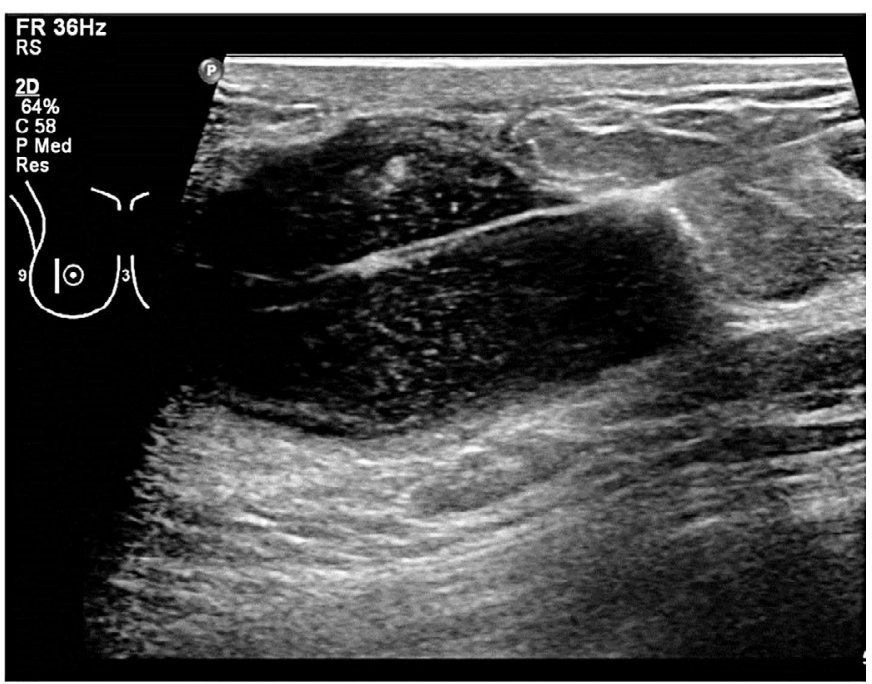

Fig. 1. A 36-year-old woman with puerperal abscess 12 weeks after delivery. Breast sonogram shows $5 \times 3 \mathrm{~cm}$ slightly lobulated, hypoechoic collections with posterior enhancement within the right lower outer quadrant and inserted needle for aspiration. clinical diagnosis of an abscess was made when compatible sonographic findings were observed in the clinical setting of mastitis. Breast abscesses were considered lactational if the subject was breastfeeding or immediately postpartum at the time of presentation, and a total of 74 patients were determined to have had lactational breast abscesses. The subset of those patients who underwent needle aspiration or VAB to treat a lactational breast abscess at our institution was included in the study. The decision of whether to perform needle aspiration cytology or VAB was made at the discretion of the performing radiologists. Thirty patients were treated with antibiotics alone, and the remaining 44 patients underwent needle aspiration (25 patients) or VAB (19 patients) The patients' medical records and imaging findings were reviewed to obtain information regarding the abscess size, microbiology findings, the number of interventions, the cure rate, and the healing time, and we compared those data between the two groups. The cure rate was defined to include patients who did not require further invasive treatment, such as incision and drainage or repeat drainage procedures, in the same breast quadrant within 6 months. Healing time was defined as the time from the initial procedure to the patient's last visit without symptoms. All statistical analyses were performed using SPSS ver. 11.0 (SPSS Inc., Chicago, IL, USA). We used the Student's t test and the chi-square test to compare the variables. For all analyses, results were considered statistically significant if the P-value was $\leq 0.05$.

\section{Needle Aspiration}

All aspiration procedures were performed under ultrasonography guidance with local anesthesia using $2 \%$ lidocaine. A straight needle (16-20 gauge) attached to a syringe was inserted into the abscess, and as much fluid as possible was aspirated (Fig. 1). We most commonly used 18-gauge needles. The aspirated fluid was sent to microbiology for Gram staining, aerobic and anaerobic culture, and sensitivity. Aspiration was repeated every other day until the lesion completely resolved.

\section{Vacuum-Assisted Biopsy}

General anesthesia was administered in two patients with multiple lesions and severe pain. Local anesthesia was selected by all other patients. We used the Mammotome (Ethicon Endo-Surgery, Cincinnati, $\mathrm{OH}, \mathrm{USA}$ ) as the VAB device. The needle was inserted into the abscess cavity away from the inflamed skin (Fig. 2) and we evacuated the abscess by vacuum until the cavity disappeared.

\section{Results}

The patients ranged in age from 19 to 42 years (mean, 30.1 years). 


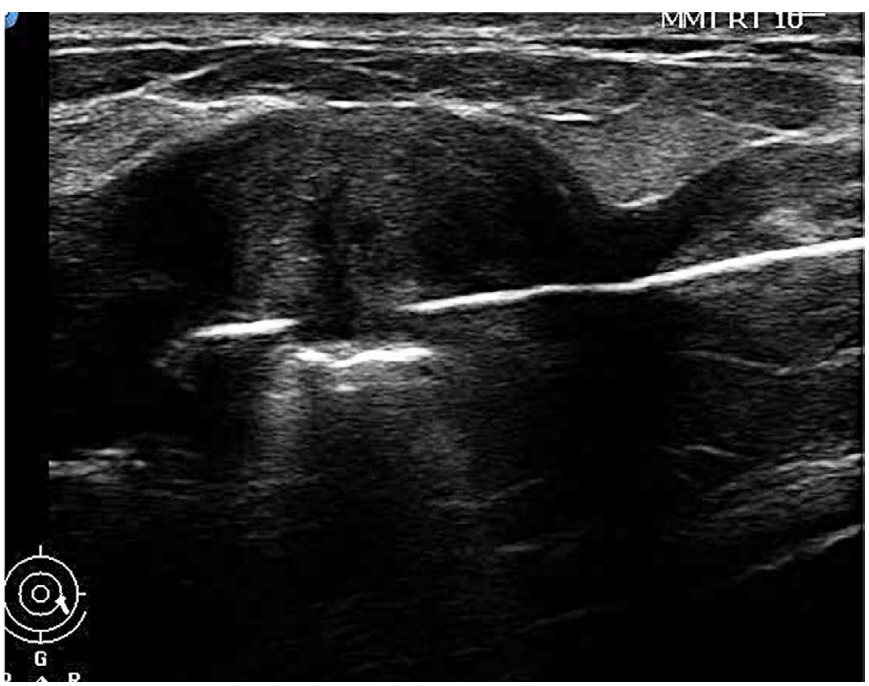

Fig. 2. A 28-year-old woman with puerperal abscess 8 weeks after delivery. Breast sonogram shows $6 \times 4 \mathrm{~cm}$ hypoechoic collection within the left lower outer quadrant and inserted vacuum-assisted biopsy needle.

The abscess size ranged from 2 to $9 \mathrm{~cm}$ (mean, $4.2 \mathrm{~cm}$ ). On average, the diagnosis of abscess was made 12.5 weeks following delivery (range, 8 to 21 weeks). The pus cultures from the breast abscesses showed Staphylococcus organisms in 24 patients (14 aureus, six coagulase-negative, and four multi-drug-resistant), beta-hemolytic Streptococcus in three patients and Pseudomonas aeruginosa in two patients. All patients were treated with antibiotics.

The patient characteristics of the needle aspiration and VAB groups are summarized in Table 1. No statistically significant differences were found between the two groups in age, the duration of lactation, and the presence of Staphylococcus organisms in the pus culture.

In the needle aspiration group, 16 patients underwent one procedure, seven underwent two procedures, and two underwent three procedures. All of the 19 patients in the VAB group underwent only one intervention. Since three patients $(12 \%)$ in the needle aspiration group and one patient (5.3\%) in the VAB group did not fully heal, incision, and drainage procedures were performed. The cure rate was not significantly different in the needle aspiration group compared to the VAB group ( $22 / 25,88 \%$ vs. $18 / 19,94.7 \%$, $\mathrm{P}=0.441)$. No recurrence took place in either group during the follow-up period. The mean healing time was significantly shorter in the VAB group (6.7 days) than in the needle aspiration group (9.0 days) $(P=0.001)$ (Table 2).

\section{Discussion}

A breast abscess, which is a painful infection caused by bacteria,
Table 1. Characteristics of the patients with breast abscess by treatment group

\begin{tabular}{lccc}
\hline \multicolumn{1}{c}{ Characteristic } & $\begin{array}{c}\text { Needle aspiration } \\
(\mathrm{n}=25)\end{array}$ & $\begin{array}{c}\text { Vacuum assisted } \\
\text { biopsy }(\mathrm{n}=19)\end{array}$ & P-value \\
\hline Age $(\mathrm{yr})$ & $30.4 \pm 4.7$ & $29.6 \pm 5.8$ & 0.613 \\
$\begin{array}{l}\text { Duration of } \\
\text { lactation (wk) }\end{array}$ & $13 \pm 2.9$ & $11.8 \pm 3.2$ & 0.217 \\
$\begin{array}{l}\text { Size of abscess (cm) } \\
\begin{array}{l}\text { Staphylococcus in } \\
\text { pus culture }\end{array}\end{array}$ & $5.1 \pm 1.4$ & $5.4 \pm 1.5$ & 0.504 \\
\hline
\end{tabular}

Values are presented as number (\%) and mean \pm SD.

Table 2. Treatment results of the patients with breast abscesses by treatment group

\begin{tabular}{lccc}
\hline \multicolumn{1}{c}{ Variable } & $\begin{array}{c}\text { Needle aspiration } \\
(\mathrm{n}=25)\end{array}$ & $\begin{array}{c}\text { Vacuum assisted } \\
\text { biopsy }(\mathrm{n}=19)\end{array}$ & P-value \\
\hline Cure rate & $22(88)$ & $18(94.7)$ & 0.441 \\
Healing time (day) & $9.0 \pm 2.4$ & $6.7 \pm 1.2$ & 0.001 \\
\hline
\end{tabular}

Values are presented as number (\%) and mean \pm SD.

is the accumulation of pus in a localized area of the breast. Lactational breast abscesses are a troublesome problem related to breastfeeding. The most common pathogen is $S$. aureus, which gains entry from the nursing mother's skin flora or from the mouth of the nursing infant $[9,10]$. Clinical assessment can be inaccurate, and an abscess located deep in the breast may be difficult to detect clinically. Ultrasonography is very useful imaging modality in the detection of breast abscesses in cases of complicated mastitis. Therefore, if a breast abscess is suspected, or if mastitis does not respond to treatment, ultrasonography should be performed [11].

Pus removal is the basic principle of medical intervention once a breast abscess is formed. Therefore, the traditional firstline treatment of breast abscesses is incision and drainage with antibiotic therapy. However, this can lead to prolonged healing time, difficulties in breastfeeding, and unsatisfactory cosmetic outcomes $[2,12]$. Currently, surgical treatment is typically reserved for recurrent or extremely large abscesses. The current first-line treatment for most abscesses is needle aspiration with antibiotics $[3,4]$. Needle aspiration for the treatment of breast abscesses has been validated as an effective treatment method $[13,14]$. Several studies have shown that ultrasound-guided treatments such as ultrasound-guided needle aspiration and ultrasound-guided catheter placement are safe, well-tolerated, and successful means of treating breast abscesses in lactating women [15-17]. However, needle aspiration has clear limitations for large-sized abscesses, abscesses that have been present for a relatively long duration at the time of presentation, multiloculated abscesses, and abscesses with an 
unusually thick wall. With the development of minimally invasive breast biopsy systems, large-bore ultrasound-guided VAB has emerged in recent years as a safe and cost-effective alternative to open surgery for the removal of certain benign breast lesions such as nonpalpable breast lesions and benign fibroadenomas $[18,19]$. Recently, it has been suggested as a new strategy for the treatment of breast abscesses $[7,8]$. Since the VAB probe is much thicker than the aspiration needle and it uses negative pressure, VAB can suck out the contents of larger and multiloculated abscesses more easily than fine needle aspiration. Some studies have reported successful outcomes of VAB in breast abscesses [7,8]. Wang et al. [8] also suggested that the Mammotome system (Ethicon Endo-Surgery) can be used in combination with appropriate antibiotic therapy as the first-line approach to breast abscesses in patients who have experienced needle aspiration failure or whose abscesses involve a large diameter $(3.5 \mathrm{~cm})$, skin inflammation, or multilocularity and viscous pus [8].

However, no previous studies have compared VAB and needle aspiration for the treatment of lactational breast abscesses. The present study showed that the cure rate in the VAB group was not inferior to that of the needle aspiration group. With respect to healing time, the VAB group showed a significantly shorter mean duration (6.7 \pm 1.2 days) than the needle aspiration group $(9.0 \pm 2.4$ days). No significant difference was found in the median size of the abscesses between the needle aspiration group $(5.1 \pm 1.4 \mathrm{~cm})$ and the VAB group $(5.4 \pm 1.5 \mathrm{~cm})$. When the size of the abscesses was similar, the VAB probe, which is thicker than the aspiration needle, could suck out of the contents much more easily. In addition, in breast abscesses with a large area of inflammation, it is difficult to choose the injection site for needle aspiration due to the short needle length. With the longer VAB probe, we were also able to easily treat abscesses with larger areas of inflammation.

We suggest that it is not necessary to use the relatively expensive VAB for all breast abscesses. Several studies have demonstrated that more than $90 \%$ of lactating abscesses and $80 \%$ of nonlactational abscesses resolved after needle aspiration with appropriate antibiotics $[20,21]$. However, considering the shorter healing time observed in the VAB group in comparison with the needle aspiration group, VAB may be used in cases of large or multiloculated abscesses.

Our study has some limitations. First, this design of this study was retrospective and observational, and our study population was relatively small. Second, the patient population of our study was limited to only lactational breast abscesses. Since the success rate of treating lactational breast abscesses is higher than for other breast abscesses, our results are inapplicable to other breast abscesses. Third, we did not compare the economic efficiency of these two methods, although VAB is known to be much more expensive. Finally, we did not investigate the continued breastfeeding rate, which is an important consideration in the treatment of lactational breast abscesses. Despite these limitations, the data from our study do demonstrate the clinical safety of VAB for lactational breast abscesses, showing an equivalent cure rate and a shorter healing time than needle aspiration.

In conclusion, VAB is a viable option for the management of lactational breast abscesses and was found to be associated with a shorter healing time than was observed in patients who underwent needle aspiration. However, further study is necessary to establish the clinical efficacy of vacuum-assisted biopsy in the management of lactational breast abscesses.

ORCID: Yun-Dan Kang: http://orcid.org/0000-0003-1411-5485; You Me Kim: http://orcid.org/0000-0001-5807-9012

\section{Conflict of Interest}

No potential conflict of interest relevant to this article was reported.

\section{Acknowledgments}

This study was supported in part by the Research Fund of the Korean Society of Ultrasound in Medicine.

\section{References}

1. Trop I, Dugas A, David J, El Khoury M, Boileau JF, Larouche N, et al. Breast abscesses: evidence-based algorithms for diagnosis, management, and follow-up. Radiographics 2011;31:1683-1699.

2. Benson EA. Management of breast abscesses. World J Surg 1989;13:753-756.

3. Schwarz RJ, Shrestha R. Needle aspiration of breast abscesses. Am J Surg 2001;182:117-119.

4. Karstrup S, Solvig J, Nolsoe CP, Nilsson P, Khattar S, Loren I, et al. Acute puerperal breast abscesses: US-guided drainage. Radiology 1993;188:807-809.

5. Eryilmaz R, Sahin M, Hakan Tekelioglu M, Daldal E. Management of lactational breast abscesses. Breast 2005;14:375-379.

6. Nakano S, Sakamoto H, Ohtsuka M, Mibu A, Sakata H, Yamamoto M. Evaluation and indications of ultrasound-guided vacuum-assisted core needle breast biopsy. Breast Cancer 2007;14:292-296.

7. Varey AH, Shere MH, Cawthorn SJ. Treatment of loculated lactational breast abscess with a vacuum biopsy system. Br J Surg 2005:92:1225-1226.

8. Wang $K$, Ye Y, Sun $G$, Xu Z. The Mammotome biopsy system is an effective treatment strategy for breast abscess. Am J Surg 2013:205:35-38.

9. Marchant DJ. Inflammation of the breast. Obstet Gynecol Clin 
North Am 2002;29:89-102.

10. Marshall BR, Hepper JK, Zirbel CC. Sporadic puerperal mastitis: an infection that need not interrupt lactation. JAMA 1975;233:13771379.

11. Dener $C$, Inan A. Breast abscesses in lactating women. World J Surg 2003;27:130-133.

12. Dixon JM. Repeated aspiration of breast abscesses in lactating women. BMJ 1988;297:1517-1518.

13. Thirumalaikumar $S$, Kommu S. Best evidence topic reports: aspiration of breast abscesses. Emerg Med J 2004;21:333-334.

14. Smith T. Non-operative management of breast abscess. N Z Med J 2009;122:95-96.

15. Hook GW, Ikeda DM. Treatment of breast abscesses with USguided percutaneous needle drainage without indwelling catheter placement. Radiology 1999;213:579-582.

16. Ulitzsch D, Nyman MK, Carlson RA. Breast abscess in lactating women: US-guided treatment. Radiology 2004;232:904-909.
17. Giess CS, Golshan M, Flaherty K, Birdwell RL. Clinical experience with aspiration of breast abscesses based on size and etiology at an academic medical center. J Clin Ultrasound 2014;42:513-521.

18. Chen SC, Yang HR, Hwang TL, Chen MF, Cheung YC, Hsueh S. Intraoperative ultrasonographically guided excisional biopsy or vacuum-assisted core needle biopsy for nonpalpable breast lesions. Ann Surg 2003;238:738-742.

19. Grady I, Gorsuch H, Wilburn-Bailey S. Long-term outcome of benign fibroadenomas treated by ultrasound-guided percutaneous excision. Breast J 2008;14:275-278.

20. O'Hara RJ, Dexter SP, Fox JN. Conservative management of infective mastitis and breast abscesses after ultrasonographic assessment. Br J Surg 1996;83:1413-1414.

21. Christensen AF, Al-Suliman N, Nielsen KR, Vejborg I, Severinsen $\mathrm{N}$, Christensen $\mathrm{H}$, et al. Ultrasound-guided drainage of breast abscesses: results in 151 patients. Br J Radiol 2005;78:186-188. 